\section{AB0881 COMPARISION OF FUNCTIONAL OUTCOMES BETWEEN PRIMARY AND REVISION TOTAL KNEE ARTHROPLASTY}

\section{K. Sevik ${ }^{1}$, T. Ünver ${ }^{1}$, B. Unver ${ }^{1} .{ }^{1}$ Dokuz Eylul University, Izmir, Turkey}

Background: Total knee arthroplasty (TKA) is the gold-standard treatment for end-stage knee osteoarthritis (OA). An increase in the prevalance of primary and revision TKA is projected due to aging of the population, increase in the obesity and OA prevalence, patients' quality of life perceptions and primary TKA procedures. Although TKA reliably improves pain and function; gait ability and function are still low compared to normal levels [1]. It is important to understand the prognosis to decide to undergo a rTKA (revision total knee arthroplasty) or enhance treatment protocols [2].

Objectives: The aim of the study is to compare the functional results of primary and revision TKA.

Methods: Hospital Of Special Surgery knee score (HSS), The Figure-Of-8 Walk Test (F8WT), The Modified Four Step Square Test (mFSST) and the 3-Meter Backwards Walk Test (3MBWT) were used for function assessment.

Table 1. Characteristics of the patients

\begin{tabular}{lccc}
\hline & TKA & rTKA & $p$ \\
\hline HSS & $83.00(74.00-90.00)$ & $78.50(68.75-90.25)$ & 0.179 \\
Age-years & $65.00(57.00-70.25)$ & $69.00(59.50-75.75)$ & 0.178 \\
BMl-kg/cm & $30.9028 .41-34.65)$ & $31.61(25.54-36.41)$ & 0.748 \\
Time after surgery-years & $2.00(1.50-4.25)$ & $3.00(2.00-6.50)$ & 0.038 \\
Female & $25(80.6 \%)$ & $20(69.0 \%)$ & 0.296 \\
Male & $6(19.4 \%)$ & $9(31.0 \%)$ & \\
Pain & $0.00(0.00-0.00)$ & $0.00(0.00-0.75)$ & 0.127 \\
${ }^{*} \mathrm{p}<0.05$ & & &
\end{tabular}

Table 2. Functional Performances Of The Patients

\begin{tabular}{lccc}
\hline & TKA & rTKA & $P$ \\
\hline 3MBWT & $6.2(3.80-8.69)$ & $7.68(6.10-11.25)$ & $\mathbf{0 . 0 4 2}^{*}$ \\
mFSST & $10.20(9.00-12.98)$ & $13.10(11.25-15.07)$ & $\mathbf{0 . 0 0 1}^{*}$ \\
F8WT & $6.23(4.74-8.6)$ & $9.11(7.15-12.05)$ & $\mathbf{0 . 0 0 1}^{*}$ \\
\hline
\end{tabular}

Conclusion: Functional status, fall risk, balance and walking skills of the rTKA patients were lower than the TKA patients. rTKA patients experience longer operation time, hospital stay and make fewer functional gains. Improvement after rTKA is also reported to be lower than TKA and balance could be worsened or does not improve after TKA [2].

Walking skills of the rTKA patients were worse than the TKA patients which may cause rTKA patients to be more cautious and tentative due to fear of falling and failure of the implant leading a more impaired function [2]. rTKA patients' balance was lower and had more fall risk than the patients with TKA. These may be due to the recurrent incision of soft tissues causing a loss of more mechanoreceptors and a greater impairment of proprioception. These findings can help clinicians to make a more informed decision for both primary and revision procedures [3]. References:

[1] Jiang Y, Sanchez-Santos MT, Judge AD et al. Predictors of Patient-Reported Pain and Functional Outcomes Over 10 Years After Primary Total Knee Arthroplasty: A Prospective Cohort Study. J Arthroplasty 2017; 32: 92-100.e102.

[2] Vincent KR, Vincent HK, Lee LW, Alfano AP. Inpatient rehabilitation outcomes in primary and revision total knee arthroplasty patients. Clin Orthop Relat Res 2006; 446: 201-207.

[3] Wodowski AJ, Swigler CW, Liu H et al. Proprioception and knee arthroplasty: a literature review. Orthopedic Clinics 2016; 47: 301-309.

Disclosure of Interests: None declared

DOI: 10.1136/annrheumdis-2020-eular.4336

\begin{tabular}{|l|l}
\hline AB0882 & RELIABILITY OF THE 3-M BACKWARDS WALK \\
TEST IN PATIENTS WITH REVISION TOTAL KNEE \\
ARTHROPLASTY
\end{tabular}

B. Unver ${ }^{1}, \underline{\text { K. Sevik }}{ }^{1}$, V. Karatosun ${ }^{2} .{ }^{1}$ Dokuz Eylul University, Izmir, Turkey; ${ }^{2}$ Dokuz Eylul University Hospital, Izmir, Turkey

Background: Total knee arthroplasty (TKA) is an effective treatment for patients suffering from end-stage osteoarthritis with 10- to 15-year implant survivorship rates exceeding $90 \%$. Infection, osteolysis due to polyethylene wear, loosening, stiffness and instability may cause to implant failure and revision of the TKA (rTKA). However, up to $20 \%$ of patients continue to have postoperative pain, functional limitations and low treatment satisfaction and $24 \%$ of patients were not able to walk unaided with no limp or a slight limp after TKA [1].

Falls and related traumas can produce "fracture, dislocation, crushing, and other injuries" [2]. Falls often occur due to impaired physical function which includes changes in lower knee joint angle, weakened lower limb muscles, peripheral nerve blockade, deformity of the foot limited balance and gait.

Several measures of fall risk have been previously developed however, recent research has demonstrated that backwards walking is more sensitive at identifying changes in mobility and balance compared to forward walking. Backwards walking is necessary to perform such tasks as backing up to a chair, opening up a door or getting out of the way of a sudden obstacle.

3-M Backwards Walk Test (3MBWT) is used to evaluate walking skills, fall risk and dynamic balance. The 3MBWT demonstrated similar or better diagnostic accuracy for falls in the past year than the most commonly used measures and found to be reliable in healthy subjects. [3] However, its reliability in rTKA has not been investigated.

Objectives: The purposes of this study were to determine the test-retest reliability and the minimal clinically important difference (MCID) of the 3MBWT in patients with rTKA

Methods: Twenty-two patients with rTKA, operated on by the same surgeon, were included. For the 3MBWT, a distance of 3 meters was marked with tape and participants were asked to align their heels with the black tape. They were instructed to walk backwards as quickly. Patients performed trials for 3MBWT twice on the same day. Between the trials, patients waited for an hour on sitting position to prevent fatigue.

Results: The 3MBWT showed an excellent test-retest reliability. Intraclass correlation coefficient ICC for 3MBWT was 0.97 . The standard error of measurement and MCID at the 95\% confidence level for 3MBWT were 1,08 and 2,99 respectively.

Conclusion: The 3MBWT has an excellent test-retest reliability in patients with rTKA. It is an effective and reliable tool for measuring fall risk, dynamic balance and walking skills. As a clinical test, the 3MBWT is easy to score, has no cost, needs no special equipment and can be applied in a short time as part of the routine medical examination.

\section{References:}

[1] Shan L, Shan B, Suzuki A et al. Intermediate and long-term quality of life after total knee replacement: a systematic review and meta-analysis. JBJS 2015; 97: 156-168.

[2] Johnson RL, Duncan CM, Ahn KS et al. Fall-Prevention Strategies and Patient Characteristics That Impact Fall Rates After Total Knee Arthroplasty. Anesthesia \& Analgesia 2014; 119: 1113-1118.

[3] Carter V, Jain T, James $\mathrm{J}$ et al. The 3-m Backwards Walk and Retrospective Falls: Diagnostic Accuracy of a Novel Clinical Measure. J Geriatr Phys Ther 2017 Disclosure of Interests: None declared

DOI: 10.1136/annrheumdis-2020-eular.5198

\section{AB0883 RELIABILITY OF THE MODIFIED FOUR SQUARE STEP TEST IN PATIENTS WITH REVISION TOTAL KNEE ARTHROPLASTY}

B. Unver ${ }^{1}$, K. Sevik ${ }^{1}$, V. Karatosun ${ }^{2} .{ }^{1}$ Dokuz Eylul University, Izmir, Turkey;

${ }^{2}$ Dokuz Eylul University Hospital, Izmir, Turkey

Background: Patients with total knee arthroplasty (TKA) often experience pain and reduced balance control, which may predispose them to greater fall risk. The patients with revision total knee arthrooplasty (rTKA), have more pain, stiffness and physical dysfunction and less postoperative improvement compared to the patients with TKA [1]. Falls in people with gait or balance disorders have significant consequences. Fear of falling can also predispose people to inactivity, which can lead to problems of debilitation, increased handicap, and disability by itself. Most of the falls take place in the course of movement, and the trips and slips were determined as the most common cause of elderly falls. Trips are responsible of falls between $40 \%$ to $60 \%$ and slips between $10 \%$ to $15 \%$, showing that the capability to take a quick step would prevent many falls [2]. Literature has found stepping speed to the different directions declines with aging and are lesser for fallers than for nonfallers [3].

Modified four square step test (mFSST) was developed to assessing fall risk and dynamic balance by scoring time while participants stepping in multiple directions but its reliability has not been investigated in patients undergoing rTKA.

Objectives: The aims of this study were to determine the test-retest reliability and the minimal clinically important change (MCID) of the mFSST in patients with rTKA 
Methods: mFSST administered on 22 patients undergoing rTKA. mFSST is performed by using tapes to make one horizontal and one vertical line like a cross to create 4 quadrants. Patients' performances were timed as patients were successfully stepping clockwise and counter-clockwise while avoid touching on tapes, turning their body or losing balance. Two trials performed and patients rested between trials and were encouraged to rest as often as they required to prevent fatigue. Results: ICC ${ }_{(2.1)}$ for mFSST was 0.83 . The standard error of measurement and MCID were 0.67 and 1.85 respectively ( $95 \%$. confidence level).

Conclusion: The mFSST has a good test-retest reliability in patients with rTKA. It is a reliable and responsive tool for measuring fall risk, dynamic balance and mobility. The mFSST is an excellent measure of gait variability, stepping in multipl directions and dynamic balance, also can easily identify real clinically important changes in patients with rTKA in simple environments and minimal equipment. References:

[1] Järvenpää J, Kettunen J, Miettinen H, Kröger H. The clinical outcome of revision knee replacement after unicompartmental knee arthroplasty versus primary total knee arthroplasty: 8-17 years follow-up study of 49 patients. International Orthopaedics 2010; 34: 649-653.

[2] Cumming RG, Klineberg RJ. Fall frequency and characteristics and the risk of hip fractures. Journal of the American Geriatrics Society 1994; 42: 774-778.

[3] Medell JL, Alexander NB. A clinical measure of maximal and rapid stepping in older women. The Journals of Gerontology Series A: Biological Sciences and Medical Sciences 2000; 55: M429-M433.

Disclosure of Interests: None declared

DOI: 10.1136/annrheumdis-2020-eular.5494

\section{AB0884 METABOLIC UNHEALTHY PHENOTYPE OF OBESITY IN PATIENTS WITH KNEE OSTEOARTHRITIS: THE EFFECTIVENESS OF ORLISTAT.}

E. Strebkova ${ }^{1}$, L. Alekseeva ${ }^{1} .{ }^{1}$ Federal State Budgetary Scientific Institution "Scientific Research Institute of Rheumatology named after V.A. Nasonova", Moscow, Russian Federation

Background: Obesity is an important socio-medical problem of mankind. Since the number of obese people in the world is increasing by about $1 \%$ per year, the immediate prospects do not look optimistic One of the important risks of the development and progression of osteoarthritis (OA) is the metabolically unhealthy phenotype of obesity, which is accompanied by a violation of lipid and carbohydrate metabolism, a violation of cytokine regulation. An important step in the treatment of patients with obesity and OA of the knee joints is weight loss.

Objectives: To evaluate the effectiveness of complex therapy of metabolic unhealthy phenotypes of obesity using orlistat (an intestinal lipase inhibitor) in the clinical manifestations of knee OA, dynamic markers of lipid and carbohydrate metabolism, dynamic CRP and leptin

Methods: The study included 50 female patients with knee OA Kellgren-Lawrence stage II-III and obesity (body mass index $(\mathrm{BMI})>30 \mathrm{~kg} / \mathrm{m}^{2}$ ), aged $45-65$ yo. Group 1 ( 25 patients) was administered orlistat at $120 \mathrm{mg}$ (1 capsule) 3 times a day for 6 month combined with low-calorie diet and therapeutic physical exercise. Group 2 (25 patients) was administered only life-modifying therapy for 6 month. The clinical parameters of the course of knee OA (WOMAC), an assessment of the quality of life (EQ-5D) were assessed at initially and after 6 months. Also initially and after 6 months a laboratory study of peripheral blood was performed glucose, HDL, LDL, TG, CRP, leptin, and the index of visceral obesity (IVO) was calculated.

Results: Initially, all patients had signs of a metabolically unhealthy phenotype of obesity: waist circumference (WC) $>88 \mathrm{~cm}$, high IVO, dyslipidemia, arterial hypertension, hyperleptinemia. After 6 months of complex therapy of obesity using orlistat in patients of group 1, a significant decrease in body weight by $10.07 \%(p<0.05)$, a decrease in WC by $8 \mathrm{~cm}(p<0.05)$, a significantly significant decrease were achieved IVO $(p<0.05)$, glucose $(p<0.05)$. Analysis of cytokines showed a significant decrease in leptin $(p<0.05)$ and CRP $(p<0.05)$ in patients with a weight loss of more than $10 \%$. The reduction in body weight in group 1 patients reduced pain by $52 \%(p<0.05)$, stiffness by $51 \%(p<0.05)$, improved joint functional failure by $51 \%(p<0.05)$ and improved quality life by $52 \%$ ( $p$ $<0.05$ ). In patients of group 2 , against the background of non-drug therapy of obesity, body weight decreased by $0.84 \%$ ( $p>0.05)$, slightly decreased WC ( $p>$ 0.05). Indicators of lipid metabolism, IVO, glucose, leptin remained unchanged ( $p>0.05$ ). The increase in CRP in patients of the 2nd group was determined by $24.54 \%(p<0.05)$.

Conclusion: The results of our study demonstrate the effectiveness of the use of a comprehensive drug regimen for the treatment of metabolically unhealthy phenotype of obesity for the clinical manifestations of osteoarthritis of the knee joints; it contributes to the improvement of metabolic parameters: a decrease in WC, lipid and carbohydrate metabolism, CRP and leptin. Thus, the use of complex therapy using orlistat in patients with osteoarthritis and obesity leads to a decrease in the metabolic activity of adipose tissue, helps to reduce cardiometabolic risk and to improve the clinical course of osteoarthritis.

Disclosure of Interests: None declared

DOI: 10.1136/annrheumdis-2020-eular.6292

\begin{tabular}{|l|l}
\hline AB0885 & EFFECTIVENESS AND SAFETY OF GLUCOSAMINE \\
& AND CHONDROITIN COMBINATION IN PATIENTS WITH \\
& KNEE AND HIP OSTEOARTHRITIS: INTERIM ANALYSIS \\
& RESULTS OF AN OBSERVATIONAL STUDY
\end{tabular}

K. Telyshev ${ }^{1}$, L. Alekseeva ${ }^{1}$, A. Lila ${ }^{2}$, A. Baranov ${ }^{3}$, E. Trofimov ${ }^{4} .{ }^{1}$ Scientific Research Institute of Rheumatology named after V.A. Nasonova, Laboratory of Osteoarthritis, Moscow, Russian Federation; ${ }^{2}$ Scientific Research Institute of Rheumatology named after V.A. Nasonova, Moscow, Russian Federation; ${ }^{3}$ Yaroslavl State Medical University, Yaroslavl, Russian Federation; ${ }^{4}$ North-Western State Medical University named after I.I. Mechnikov, Saint Petersburg, Russian Federation

Background: Combined treatment with oral glucosamine hydrochloride $(\mathrm{GH})$ and chondroitin sulfate (CS) was shown to be efficient for pain relief and function improvement in osteoarthritis patients with moderate to severe knee pain $[1,2]$

Objectives: To investigate demographic and clinical characteristics, changes in pain, functions of daily living, quality of life and treatment satisfaction of patients with knee osteoarthritis (KOA) or hip osteoarthritis ( $\mathrm{HOA}$ ) receiving long-term treatment with oral $\mathrm{GH}$ and $\mathrm{CS}$ combination in routine clinical practice.

Methods: An open-label, multicenter, observational prospective study is being conducted in the Russian Federation. Patients of both sexes with KOA or HOA (Kellgren and Lawrence grades I-III) who receive GH 500 mg+CS 400 mg capsules three times a day for the first 3 weeks of treatment, then twice daily, are included in the study. The interim analysis has been conducted after the first 550 enrolled patients (50\% of the total sample size) had completed the first follow-up visit (Week 16-24 after the start of treatment).

Results: The study group included 406 (73.8\%) patients with KOA and 144 (26.2\%) patients with HOA enrolled in 43 centers in Russia. The mean age of the patients was 61.1 years; most patients were women $(88.7 \%)$. The predominant risk factors for OA were non-genetic causes (excess weight, hormonal disorders, malformations of bones and joints, joint operations) (52.8\% of patients) exogenous risk factors (professional activity, trauma, sports) and hereditary diseases of bones and joints were reported in $15.5 \%$ and $4.1 \%$ of patients respectively.

Interim analysis showed clinically significant improvement in each of the KOOS and HOOS subscales at 4-6 months after the start of treatment. In patients with KOA, the mean score increase was 15.7 for the Pain subscale, 14.6 for the Quality of Life subscale, 13.8 for the Physical function (KOOS-PS), and 11.7 for the Symptoms subscale. The percentage of patients who rated the pain frequency as 'always' or 'daily' decreased from $60 \%$ to $25 \%$.

In patients with HOA, the mean score increase was 16.0 for the Pain subscale, 14.3 for the Quality of Life subscale, 16.1 for the Physical function (HOOS-PS), and 10.3 for the Symptoms subscale. The percentage of patients who rated the pain frequency as 'never' or 'monthly' increased from 34\% to $60 \%$.

Most patients $(89.1 \%)$ were receiving the medicinal product for $\geq 3$ months. Treatment-related AEs were reported in $16(3.0 \%)$ patients and mainly included gastrointestinal tract disorders (in $12(2.2 \%)$ patients)

Conclusion: The results obtained at 4-6 months after the start of treatment demonstrate clinically significant reduction of frequency and intensity of pain and other OA symptoms, as well as improvement of functions in daily living and quality of life in patients with KOA or HOA after the first course of treatment with $\mathrm{GH}+$ CS capsules. The majority of patients $(72.5 \%)$ were satisfied with the treatment The incidence of drug-related AEs was low, and the nature of AEs was consistent with known safety profile of $\mathrm{GH}$ and CS combination.

\section{References:}

[1] Clegg DO, Reda DJ, Harris CL, et al. Glucosamine, chondroitin sulfate, and the two in combination for painful knee osteoarthritis. N Engl J Med. 2006 Feb 23;354(8):795-808.

[2] Hochberg MC, Martel-Pelletier J, Monfort J, et al. Combined chondroitin sulfate and glucosamine for painful knee osteoarthritis: a multicentre, randomised, double-blind, non-inferiority trial versus celecoxib. Ann Rheum Dis. 2016 Jan;75(1):37-44

Disclosure of Interests: Kirill Telyshev: None declared, Ludmila Alekseeva Grant/research support from: Bayer, Alexander Lila: None declared, Andrey Baranov Grant/research support from: Bayer, Evgeny Trofimov Grant/research support from: Bayer

DOI: 10.1136/annrheumdis-2020-eular.3079 